\title{
Empatía histórica y aumento de la tolerancia hacia la figura de los refugiados: estudio con profesorado en formación
}

\author{
María Belén San Pedro-Veledo ${ }^{*}$ e Inés López-Manrique \\ Universidad de Oviedo
}

\begin{abstract}
Resumen. Los objetivos del estudio son, comprobar si la realización de un ejercicio de empatía histórica sobre los exiliados de la Guerra Civil contribuye al desarrollo de actitudes tolerantes hacia la figura de los refugiados en el alumnado del Grado de Maestro en Educación Primaria, y constatar la influencia de los medios de comunicación en las actitudes de los mismos hacia la figura de los refugiados y si esta puede o no modificarse. Se ha utilizado un cuestionario de elaboración propia que mide las actitudes de 79 estudiantes hacia la figura de los refugiados en cinco dimensiones: afectiva, religión, económico-laboral, social y política de inmigración. El cuestionario se cubrió en dos momentos distintos, antes y después de realizar un ejercicio de empatía histórica sobre los exiliados de la Guerra Civil Española. Los estadísticos utilizados han sido Alfa de Cronbach, Coeficiente de Correlación de Pearson, $t$ de Student, Análisis de Varianza (ANOVA) y $d$ de Cohen. Los resultados muestran que existen actitudes más positivas en los estudiantes hacia los refugiados tras realizar la práctica. Asimismo, hay diferencias estadísticamente significativas en la influencia de los medios de comunicación sobre las actitudes de los estudiantes hacia los refugiados entre pre y post intervención.
\end{abstract}

Palabras clave: Empatía histórica, Toma de perspectiva histórica, Medios de Comunicación, Actitudes, Refugiados.

Historical empathy and increased tolerance towards the figure of refugees: a study with students of teaching.

Abstract. This paper has two objectives, a) to verify if the exercises of historical empathy on the exiles of the Civil War can contribute to the development of tolerant attitudes towards the figure of the refugees in the students of the Bachelor's Degree in Primary Education Teaching, and b) confirm the influence of the mass media in the attitudes of the students towards the figure of the refugees and whether this can be changed. It has been used a specifically designed questionnaire to measure the attitudes of 79 students towards refugees in five dimensions: affection, religion, economiclabor, social and immigration policy. The questionnaire was covered in two different moments, before and after doing an exercise of historical empathy about the exiles of the Spanish Civil War. The results obtained by Cronbach alpha statistic, Pearson's correlation, Student's t, Cohen's $d$ and Analysis of Variance (ANOVA) indicate the existence of more positive students' attitudes towards refugees after the practice. Likewise, there are statistically significant differences between the first and second questionnaires in the influence of the media on students' attitudes towards refugees.

Keywords: Historical Empathy, Historical Perspective Taking, Media, Attitudes, Refugees.

Europa está viviendo en la actualidad la mayor crisis de refugiados desde el siglo XX. El número de desplazados por causas bélicas

Recibido: 09/05/2017 - Aceptado: 01/06/2017 - Avance online: 28/06/2017

*Correspondencia: María Belén San Pedro

C/Aniceto Sela, s/n. Universidad de Oviedo

C.P: 33005, Oviedo, España.

E-mail: pedromaria@uniovi.es

San Pedro, M. B. \& López, I. (2017). Empatía histórica y aumento de la tolerancia hacia la figura de los refugiados: estudio con profesorado en formación. Revista de Psicología y Educación, 12(2), 116-128, https://doi.org/10.23923/rpye2017.12.150 crece de modo exponencial. Para una política eficaz de reasentamiento se requieren, entre otros, actitudes positivas de las sociedades de acogida hacia las personas desplazadas (Esses, Hamilton y Gaucher, 2017). La llegada de refugiados a países de acogida influye en el ámbito educativo a dos niveles. Por un lado, las escuelas deben afrontar la llegada de niños

1699-9517/@ 2017 Asociación Científica de Psicología y Educación (ACIPE). Publicado por Consejo General de Colegios Oficiales de Psicólogos, España. Este es un artículo Open Access bajo la CC BY-NC-ND licencia (http://creativecommons.org/licencias/by-nc-nd/4.0/). 
y niñas en edad escolar a países con distintas lenguas a la materna y diferente cultura; por otro lado, son necesarios docentes preparados para tratar la diversidad en el aula y comprender la difícil realidad de los nuevos estudiantes. Por supuesto, esto implica necesariamente la utilización de estrategias destinadas a la eliminación de prejuicios y actitudes xenófobas en las aulas, estableciendo puentes entre los conceptos del "yo" y del "otro".

Etxeberría, Murua, Garmendia y Etxeberría (2013), tras una investigación con estudiantes de Educación Secundaria (ESO y Bachillerato) en el País Vasco, señalan que el análisis de las opiniones del alumnado sobre los inmigrantes indica la existencia de prejuicios arraigados entre los más jóvenes. Es por eso que se hace indispensable el tratamiento de una enseñanza inclusiva y tolerante desde los primeros estadios educativos. Los currículos nacionales están siendo cada vez más cuestionados, producto del impacto de la migración y de la integración europea. Aun así, la investigación sobre distintos currículos europeos indica que materias clave en la educación intercultural como la Geografía, la Historia o la Educación para la Ciudadanía en Grecia, Alemania e Inglaterra siguen teniendo aún un carácter etnocéntrico (Faas, 2011). Algunas investigaciones sobre la inclusión de refugiados en los sistemas educativos de los países de acogida señalan que los docentes se encuentran con dificultades institucionales a la hora de la correcta integración de estos estudiantes (Roxas, 2010) e incluso a veces las políticas escolares carecen de la innovación y de los recursos necesarios para involucrar a las familias del alumnado refugiado en su educación (Isik-Ercan, 2012).

En este sentido cobran importancia distintos estudios sobre el tratamiento de la diversidad cultural tanto con alumnado de enseñanzas obligatorias como con profesorado en formación o en activo. En Texas se llevó a cabo un proyecto donde estudiantes universitarios acompañaban a las nuevas familias de refugiados reasentados en su primera visita a la escuela de sus hijos con el fin de desarrollar una conciencia cultural (Calderón y Silva, 2014). Asimismo, una investigación preliminar en escuelas inglesas y escocesas explora los conceptos de los docentes sobre la "compasión" entendida como valor educativo -en términos de justicia, ayudar a los que tienen problemas en base a nociones de igualdad y derechos humanos- para integrar a los niños y niñas solicitantes de asilo y refugiados (Arnot, Pinson y Candappa, 2009). El interés por la situación de los refugiados más jóvenes en las aulas y los posibles mecanismos de los docentes para manejar conflictos existe ya desde la pasada década (Szilassy y Árendás, 2007) e incluso la realidad de las personas desplazadas se ha hecho un hueco en los temas más tratados de la literatura infantil británica con un gran potencial como herramienta educativa en los primeros cursos escolares (Hope, 2008; Monobe y Son, 2014). Todo ello indica la necesidad de una reflexión teórica y empírica más profunda de las dificultades de la experiencia de los refugiados por parte de los docentes y de los que diseñan las políticas educativas (Zembylas, 2012) con el fin de conseguir una correcta integración de este alumnado en las aulas.

$\mathrm{Si}$ bien las políticas europeas no han conseguido avanzar en la acogida de los refugiados procedentes de diversos países en conflicto, y aún son muchos los que esperan una solución a su situación en distintos campos de refugiados fronterizos, existe una percepción de la inmigración irreal que sitúa a todos los inmigrantes como solicitantes de asilo (Blinder, 2015). Con respecto a esto, no hay que olvidar la influencia de los medios de comunicación en las actitudes de la población europea hacia la figura de los refugiados. Desde 2014 tanto la prensa escrita como la televisión, internet y las redes sociales reflejan la crisis de los refugiados desde puntos de vista diversos. Algunas investigaciones establecen que los medios de comunicación pueden tener una influencia tanto positiva como negativa en los procesos de diálogo e interacción entre la comunidad de acogida y los migrantes (Galikhuzina, Penkovtsev y Shibanova, 2016), encontrándose una relación directa entre la frecuencia y las formas de consumo de los mismos y las actitudes hacia la inmigración (Álvarez-Gálvez, 2014a; Álvarez-Gálvez, 2014b). No sólo se debe indagar sobre esta 
influencia en el conjunto de la sociedad, sino que cobra aún más importancia el análisis en el contexto educativo, con docentes y alumnado, a fin de garantizar una educación lo más alejada de prejuicios y estereotipos, y que incluya el ejercicio de la literacidad crítica para diferenciar hechos y opiniones en cuanto a la fiabilidad de los medios de comunicación y de las fuentes de información (Santisteban et al., 2016).

Concretamente, desde el ámbito de la didáctica de las Ciencias Sociales se insiste en la formación de docentes para una enseñanza libre de prejuicios, que tenga como base la vida real y como objetivos enseñar a pensar y a afrontar conflictos como parte de nuestra vida cotidiana (Santisteban 2015), con el fin de preparar a los estudiantes para su participación en una sociedad democrática (Barton y Levstik, 2008). Adultos y escolares son autores y receptores de prejuicios. Aunque las actitudes que implican prejuicios y discriminación están muy arraigadas en la edad adulta, es en la infancia y adolescencia cuando surgen plenamente, por lo que es preciso fomentar un clima de aula que promueva la equidad, la inclusión y desafíe los prejuicios (Rutland y Killen, 2015). La enseñanza debe estar basada en los valores de justicia, solidaridad y la aceptación positiva de las diferencias culturales, incluyendo una reflexión en la que el alumnado ponga en tela de juicio sus estereotipos culturales y sociales teniendo en cuenta sus propias representaciones mentales (Valls, 2009).

De este modo, es preciso trabajar dichas cuestiones tanto con el alumnado como el profesorado, incidiendo en este sentido con los docentes en formación. La didáctica de las Ciencias Sociales en general, y la didáctica de la Historia en particular pueden ser claves a la hora de acercar tanto a profesorado como a alumnado a otros parámetros culturales. Existen investigaciones, en ambos sentidos, acerca de las capacidades de alumnado y docentes para relacionar aspectos del pasado con el presente. Navarro (2015) señala que el alumnado de Bachillerato manifiesta carencias en el dominio de contenido histórico que provoca dificultades para relacionar acontecimientos de la historia pasada con situaciones políticas, económicas, sociales y culturales de la actualidad. Andreu (2016), tras una investigación realizada con estudiantes de último curso del Grado en Maestro de Educación Primaria, indica que los futuros maestros desconocen en muchos casos su historia y cultura propia lo que hace muy difícil el acercamiento a otras. No obstante, propone como solución el acercamiento por medio de la empatía a través de fuentes orales.

Algunas experiencias muestran cómo la Empatía Histórica puede utilizarse para pensar históricamente situaciones de identidades en conflicto. Sáiz (2013), a través de una práctica situada en la España medieval, señala que la recreación de la participación en la conquista cristiana como una experiencia de migración histórica en la que algunos estudiantes son hijos de inmigrantes, puede servir para introducir posibles contenidos sobre las causas de las migraciones. Con respecto a la Empatía Histórica, Explicación histórica contextualizada - HPT (historical perspective taking) son numerosos los estudios que indagan en este procedimiento como elemento clave para la comprensión histórica y la formación del pensamiento histórico (Domínguez, 2015; González, Henríquez, Pagès y Santisteban, 2009; Lee y Ashby, 2001; Lévesque, 2008; Seixas, 1996; Shemilt, 1984; Trepat, 1995; Wineburg, 2001). Este procedimiento tiene como objeto comprender los actos de las personas en el pasado, teniendo en cuenta los parámetros económicos, sociales, políticos y culturales de un momento determinado. El correcto uso de este procedimiento implica necesariamente conocer el contexto de la época o período a trabajar mediante el uso de fuentes históricas primarias y secundarias para que se realice una correcta contextualización histórica libre de anacronismos y presentismo. Cuando esta contextualización falla los estudiantes manifiestan dificultades a la hora de proyectarse en el pasado e incluso en el futuro. A este respecto, Santisteban (2010) señala los resultados pobres de una experiencia en la que los estudiantes tenían que plantear una situación ficticia sobre la inmigración que les afectaría en el futuro, al no valorar las posibles problemáticas que se generan en esas situaciones. 
La bibliografía teórica ha señalado la existencia de problemas al considerar el peso del elemento empático en este procedimiento, de ahí que actualmente se opte por los términos explicación histórica contextualizada - historical perspective taking (HPT) para referirse al mismo. No obstante, Endacott (2014) utiliza un modelo de Empatía Histórica como un constructo afectivo-cognitivo, e indica la importancia del mantenimiento del equilibrio entre el contexto histórico, la toma de perspectiva y las respuestas afectivas para un uso adecuado del procedimiento. En este sentido, Carril y Sánchez (2015) manifiestan que el aprendizaje de los contenidos históricos vinculados a estrategias de comprensión y memorización guarda una escasa relación con las habilidades empáticas del alumnado. Por lo tanto, debe utilizarse una empatía que reúna una parte afectiva al mismo tiempo que una cognitiva para poder adoptar la perspectiva del otro. Algunas experiencias demuestran la importancia de la contextualización histórica unida a este elemento afectivo a la hora de entender culturas ajenas o pasadas. Es el caso de la llevada a cabo por Burgard y Boucher (2016), durante un viaje de estudios a sitios históricos del Underground Rail Road vinculados a la segregación racial en Estados Unidos. En otro caso, el objetivo de la experiencia es el contacto con el pasado multicultural como un tipo nuevo de contacto intergrupal. El programa descrito por Stefaniak y Bilewicz (2015) familiarizaba a estudiantes polacos con el patrimonio histórico judío en los sitios en los que residían. Los estudiantes transformaron sus actitudes, manifestando un mayor conocimiento de la historia judía y la historia local, una mayor inclusión de los judíos con actitudes más positivas hacia ellos e incluso aumentó también la percepción de igualdad con respecto a otros grupos ajenos. En Colombia, con el objeto de introducir en las aulas la pluralidad y la valoración de la diferencia, se ha implementado el uso de testimonios primarios y secundarios de víctimas de la guerra (Londoño y Carvajal, 2016). Los resultados evidencian que nos solo se llega a comprender el sufrimiento de las víctimas, sino que también el alumnado adquiere contenidos sobre la configuración del conflicto, sus actores y territorios, y las causas y consecuencias del mismo.

Aunque existen instrumentos de medición para evaluar la toma de perspectiva histórica (Hartmann y Hasselhorn, 2008; Huigien, Boxtel, Grift y Holthuis, 2014) e investigaciones que miden las actitudes y la consecución de objetivos de aprendizaje de la Historia de los estudiantes (Rantala, Manninen y Berg, 2016), para el propósito de este trabajo el interés reside en el modo en el que el componente afectivo de la Empatía Histórica puede influir a la hora de reducir prejuicios hacia grupos de riesgo como los refugiados, lo cual supone una novedad. En la actualidad, es preciso realizar investigaciones y llevar a cabo experiencias de este tipo con el profesorado en formación que durante sus prácticas puede encontrarse con alumnado desplazado en las aulas.

Por otro lado, algunos estudios han tenido en cuenta la relación existente entre el factor género y la empatía histórica o HPT. Los resultados obtenidos por Yang y Huang (2011), tras aplicar el HPT Model de Hartmann y Hasserlhorn (2008), no muestran diferencias significativas por razón de género en las puntuaciones obtenidas por estudiantes de Educación Secundaria con respecto a la capacidad de la toma de perspectiva histórica. Más recientemente, Carril y Sánchez (2016) tras aplicar el cuestionario de evaluación de la empatía Índice de Reactividad Interpersonal (IRI), que incluye factores cognitivos y factores afectivos, a una muestra de 78 graduados en Historia que cursaba el Máster de Profesor de ESO y Bachillerato, F. P. y Enseñanza de Idiomas en tres universidades españolas, afirman que los resultados no revelan diferencias significativas en función del sexo, si bien observan tendencias ascendentes de los alumnos varones en ítems relacionados con la adopción de perspectiva cognitiva mientras que en el caso de las alumnas las tendencias ascendentes se refieren a los ítems relacionados con la adopción de la perspectiva afectiva. Es necesario, por tanto, comprobar si el género guarda relación con el desarrollo de las actitudes tolerantes que pueden desarrollarse mediante actividades de empatía histórica. 
Los principales objetivos de este estudio han sido, por un lado, comprobar si la realización de un ejercicio de empatía histórica sobre los exiliados de la Guerra Civil puede contribuir al desarrollo de actitudes tolerantes hacia la figura de los refugiados en el alumnado del Grado de Maestro en Educación Primaria, teniendo en cuenta el factor género como variable independiente, y por otro lado constatar la influencia de los medios de comunicación en las actitudes de los mismos estudiantes hacia la figura de los refugiados y si esta influencia se modifica tras la práctica de empatía histórica. Las hipótesis de partida son las siguientes:

1. La realización de ejercicios de empatía histórica sobre los exiliados de la Guerra Civil Española contribuye a comprender mejor la situación de los refugiados en la actualidad y a disminuir prejuicios hacia los mismos.

2. Las concepciones de los estudiantes hacia la figura de los refugiados se encuentran motivadas por la influencia de los medios de comunicación, si bien la práctica de la empatía histórica puede contribuir a matizarla.

\section{MÉTODO}

\section{PARTICIPANTES}

Los participantes en este estudio han sido 79 estudiantes de la asignatura "Desarrollo curricular de las Ciencias Sociales" perteneciente al Grado de Maestro en Educación Primaria de la Universidad de Oviedo. En cuanto al género, 31 son varones $(35,6 \%)$ y $48(64,4 \%)$ son mujeres. La media de edad se sitúa en $21,54(D T=3,265)$, yendo la horquilla desde los 19 hasta los 42 años. Se ha tenido en cuenta el tipo de centro en el que se estudió la Educación Secundaria Obligatoria y así un 65,8\% estudió en un centro público, el $27,8 \%$ en uno concertado, el $3,8 \%$ en un centro privado y un $2,6 \%$ desarrolló sus estudios tanto en centros públicos como concertados y privados a lo largo de esa etapa. En cuanto a la procedencia, el $67,1 \%$ proviene de zonas urbanas, el 20,3\% de zonas semiurbanas y el $12,7 \%$ de zonas rurales. También se han recogido variables relacionadas con la formación y la situación laboral de los progenitores de los estudiantes con el fin de comprobar si estas afectaban a su percepción en cuanto a la figura de los refugiados. Así, el $35,4 \%$ de las madres del alumnado participante han cursado Bachillerato o FP2, el 22, 8\% ESO, EGB o FP1, el $16,5 \%$ posee una diplomatura, el $15,2 \%$ una licenciatura y un $10,1 \%$ posee solo estudios de Primaria o de EGB inacabada. En cuanto a la situación laboral de las mismas el $68,4 \%$ se encuentra trabajando, un $21,5 \%$ desempleadas, un 6,3\% jubiladas y un $3,8 \%$ se sitúa en la categoría "otros".

Con respecto a los padres, el 36,7\% tiene los estudios de Bachillerato o FP2, el 35,4\% los de $\mathrm{ESO}, \mathrm{EGB}$ o FP1, el 12,7\% una licenciatura, el $10,1 \%$ ha cursado Primaria o EGB inacabada y el $5,1 \%$ una diplomatura. De estos, el $72,2 \%$ se encuentra trabajando, el 15,2\% jubilados, el $7,6 \%$ desempleado y $5,1 \%$ se sitúa en la categoría "otros".

\section{PROGRAMA DE INTERVENCIÓN}

La experiencia se llevó a cabo durante cuatro sesiones en los meses de noviembre y diciembre del curso 2015-2016. En la primera de ellas se solicitó a los estudiantes que buscasen en internet dos noticias relacionadas con los refugiados sirios, indicando su procedencia y los motivos de la elección, para comprobar la posible existencia de sesgos en la misma. A continuación, tras detallar los objetivos del estudio y garantizar el anonimato en las respuestas, se pidió al alumnado que cubriese el cuestionario. Durante la siguiente sesión se trabajó con fuentes primarias y secundarias, relativas tanto a los refugiados sirios como a los exiliados de la Guerra Civil Española, accesibles en internet (testimonios de refugiados y exiliados, documentales y webs de interés) mediante su visionado, lectura y posterior debate. Como práctica evaluable relacionada, los estudiantes realizaron en las siguientes dos sesiones una composición individual que simulaba una carta escrita por un exiliado de la Guerra Civil, utilizando los procedimientos de la empatía histórica y el uso de fuentes 
históricas primarias y secundarias. El guion de la carta estaba pautado, teniendo el alumnado que contextualizar históricamente el relato del exiliado. Cada estudiante debía adoptar el rol de una persona que se ve obligada o que toma la decisión de dejar el país en el que ha desarrollado su toda su vida. Los roles podían ser los de un niño/a, un hombre o una mujer a elección del estudiante. El alumnado podía elegir también cuáles eran las circunstancias familiares y/o laborales del personaje. Tenía que aparecer necesariamente alguna referencia a un episodio de la Guerra Civil Española que provocase que el personaje optase por la vía del exilio (batalla, avance de la contienda, posible represión por motivos ideológicos, motivos económicos y pérdida del hogar, entre otros). Los motivos que impulsaban al personaje a dejar su país de origen debían justificarse históricamente. Era obligatorio detallar las circunstancias del viaje al país elegido por el estudiante en base a datos históricos acerca de los países que fueron destino de los exiliados de la Guerra Civil, basándose en el material proporcionado en la segunda sesión o seleccionado de entre otras aportaciones que cada estudiante considerase. También tenía que aludirse al contexto en el que se encontró el personaje a la llegada del nuevo país. Se explicaría además cuál fue el destino del personaje en el exilio en base al país elegido previamente (si se elegía Francia había que tener en cuenta el contexto de la II Guerra Mundial y qué pasó con los exiliados españoles en dicho país). El trabajo, de más o menos la extensión de un folio por ambas caras, debía ser narrado en primera persona a modo de reflexión, carta, diario, o similar. En anexo tenía que señalarse la procedencia de los datos utilizados para la contextualización histórica. Esta composición fue la única actividad en la que los estudiantes tuvieron que revelar su identidad. Tras la entrega de la práctica, el alumnado tuvo que cubrir nuevamente el cuestionario.

Los resultados de la primera sesión y los detalles de la práctica pueden consultarse en otro trabajo (San Pedro y López, 2016).

\section{INSTRUMENTO}

El instrumento de medida utilizado ha sido un cuestionario de elaboración personal sobre la percepción y actitudes del alumnado hacia los refugiados sirios (PREFSIR-1) formado por tres bloques de preguntas. El bloque $A$, llamado "Actitudes", está formado por 31 variables asociadas a la percepción que tienen los estudiantes de los refugiados sirios y su situación en cuanto a cuestiones de tipo afectivo, religioso, económico-laboral, social y político. Los ítems se responden en una escala tipo Likert de 1 a 5, siendo 1 totalmente en desacuerdo y 5 totalmente de acuerdo con las cuestiones presentadas.

Se han agrupado los ítems del Bloque A sobre "Actitudes" en cinco dimensiones a las que se han denominado "Afectiva", "Religión", "Económico-laboral", "Social" y "Política de Inmigración". La dimensión "Afectiva" mide las actitudes de los estudiantes hacia los refugiados desde el punto de vista de las relaciones personales. Un ejemplo de ítem en esta dimensión sería la afirmación "A2. No me importaría tener a refugiados sirios como alumnos". La dimensión "Religión" trata de conocer el grado de aceptación del alumnado en cuanto a las distintas religiones que pueden profesar los refugiados, y si existe una tendencia que vincule el terrorismo con la religión. Un ítem incluido en la misma es el "A7. La acogida de refugiados en Europa va a provocar un crecimiento de la religión islámica en España". Bajo la dimensión "Económico-laboral" se recogen una serie de ítems relacionados con los posibles escenarios que pueden aparecer tras la llegada de los refugiados en el plano del mercado laboral y en el contexto de la crisis económica. Como ejemplo de ítem podemos resaltar la aseveración "A15. La acogida de refugiados sirios puede ser muy positiva para el mercado de trabajo laboral". La dimensión "Social" mide las percepciones del alumnado en cuanto al nivel de integración de los refugiados desde el punto de vista social y cultural. Entre los ítems que la componen, se incluye la afirmación "A22. La acogida de refugiados sirios no va a perjudicar los derechos de las mujeres europeas y/o españolas". La última 
dimensión, "Política de Inmigración", agrupa distintas variables referentes a las creencias de los estudiantes con respecto a cuál debería ser el papel de los diferentes países en cuanto a las políticas de acogida de refugiados. Como ejemplo de ítem podemos señalar la afirmación "A31. Europa y España tienen como deber hacerse cargo de aquellas personas que huyen de la guerra en sus países".

El Bloque B, llamado "Medios de Comunicación", recoge seis variables que miden la procedencia de la información que poseen los estudiantes acerca de los refugiados sirios y su respectiva frecuencia. En cuanto a los medios de comunicación se han tenido en cuenta la televisión, la prensa, distintas webs de Internet, Youtube, y redes sociales como Twitter y Facebook. También se ha usado en este caso una escala Likert de 1 a 5 siendo 1 nada y 5 mucho para conocer la percepción personal del tiempo de uso de los medios para informarse.

Por último, el Bloque $C$ está formado por preguntas sociales y personales en cuanto a edad, tipo de centro en el que se estudió la Educación Secundaria, zona de vivienda y formación y situación laboral de ambos progenitores.

\section{PROCEDIMIENTO}

La intervención e investigación se llevó a cabo con un grupo-clase de la asignatura citada, dividido en dos subgrupos durante las sesiones de prácticas. Se solicitó al alumnado participante que cubriese el cuestionario en dos momentos distintos, en la primera sesión antes de la intervención educativa y en la cuarta sesión después de la misma y de la entrega de la práctica evaluable. Los cuestionarios fueron realizados en papel y bolígrafo. La primera sesión tuvo lugar en una de las aulas de informática para que los estudiantes pudiesen buscar las noticias en línea. El resto de las sesiones se desarrollaron en el aula de prácticas ordinaria, pudiendo el alumnado también utilizar horas de trabajo personal no presencial para la elaboración de la composición. Todas las sesiones presenciales estuvieron supervisadas por el mismo docente. Con respecto a la práctica, se utilizó una rúbrica de evaluación elaborada al efecto para medir la correspondiente adecuación de cada composición al guion original proporcionado. Cabe señalar, por último, que el desarrollo de la experiencia coincidió en el tiempo con los atentados de París y de Kabul de noviembre y diciembre de 2015 respectivamente.

\section{ANÁLISIS ESTADÍSTICO}

Una vez agrupados los ítems del bloque $A$ en las cinco dimensiones explicadas anteriormente, se ha procedido al análisis descriptivo, aplicando pruebas de tendencia central y dispersión (media, desviación típica, varianza, asimetría y curtosis) así como, para comprobar si existía correlación bivariada entre las cinco dimensiones, se ha utilizado el coeficiente de Pearson.

Se han utilizado las siguientes pruebas estadísticas: Alfa de Cronbach para determinar el nivel de fiabilidad, prueba de Levene para conocer la homogeneidad de las varianzas, t de Student y Análisis de varianza (ANOVA) para el análisis de diferencias por género y la comparación de medias, y la d de Cohen para el tamaño del efecto. Con respecto a esta última se han utilizado las indicaciones de Cohen (1988), siendo $d=$ 0,20 , pequeño; $d=0,50$, medio; y $d=$ 0,80 , grande.

Para la aplicación de la ANOVA, se ha procedido a realizar un reajuste en los ítems de los medios de comunicación en tres grupos, 1 los que manifiestan que no los utilizan, 2 los que consideran que les destinan poco o algo de su tiempo, y 3 para aquellos que consideran que hacen mucho o bastante uso de los medios.

El tratamiento y análisis de los datos obtenidos se ha realizado mediante el paquete estadístico SPSS.22 para Windows (IBM, 2013) y el G*Power 3.1, programa de acceso gratuito diseñado para poder realizar estimaciones de la potencia estadística y del tamaño del efecto (Erdfelder, Faul y Buchner, 1996; Faul, Erdfelder, Lang y Buchner, 2007). 


\section{RESULTADOS}

\section{ANÁLISIS DE LAS ACTITUDES HACIA LOS REFUGIADOS PRE-TEST $Y$ POST-TEST $Y$ DIFERENCIAS POR GÉNERO}

Para conocer la fiabilidad se ha aplicado el estadístico Alfa de Cronbach. Los resultados muestran un buen ajuste en tres de las dimensiones, en las que los valores superan el ,700. Las otras dos dimensiones, aunque no alcancen el valor mínimo estipulado, están muy cerca del mismo, superando el ,660.

Al aplicar el estadístico Correlación de Pearson, para comprobar los efectos de las correlaciones bivariadas entre las cinco dimensiones, se observan valores estadísticamente significativos y en sentido positivo, en todas las correlaciones, tanto en los resultados de la primera aplicación del cuestionario como de la segunda, siendo todos valores superiores a ,300. Puede comprobarse en la Tabla 1.

En cuanto a las pruebas de tendencia y dispersión, tanto en la primera aplicación del cuestionario como en la segunda, la dimensión puntuada más alta fue la "Afectiva", mientras que la de menor puntuación fue la dimensión "Religión". La muestra presenta buena asimetría y curtosis, no llegando a alcanzar en ningún caso el valor 1,00. Ver Tabla 2.

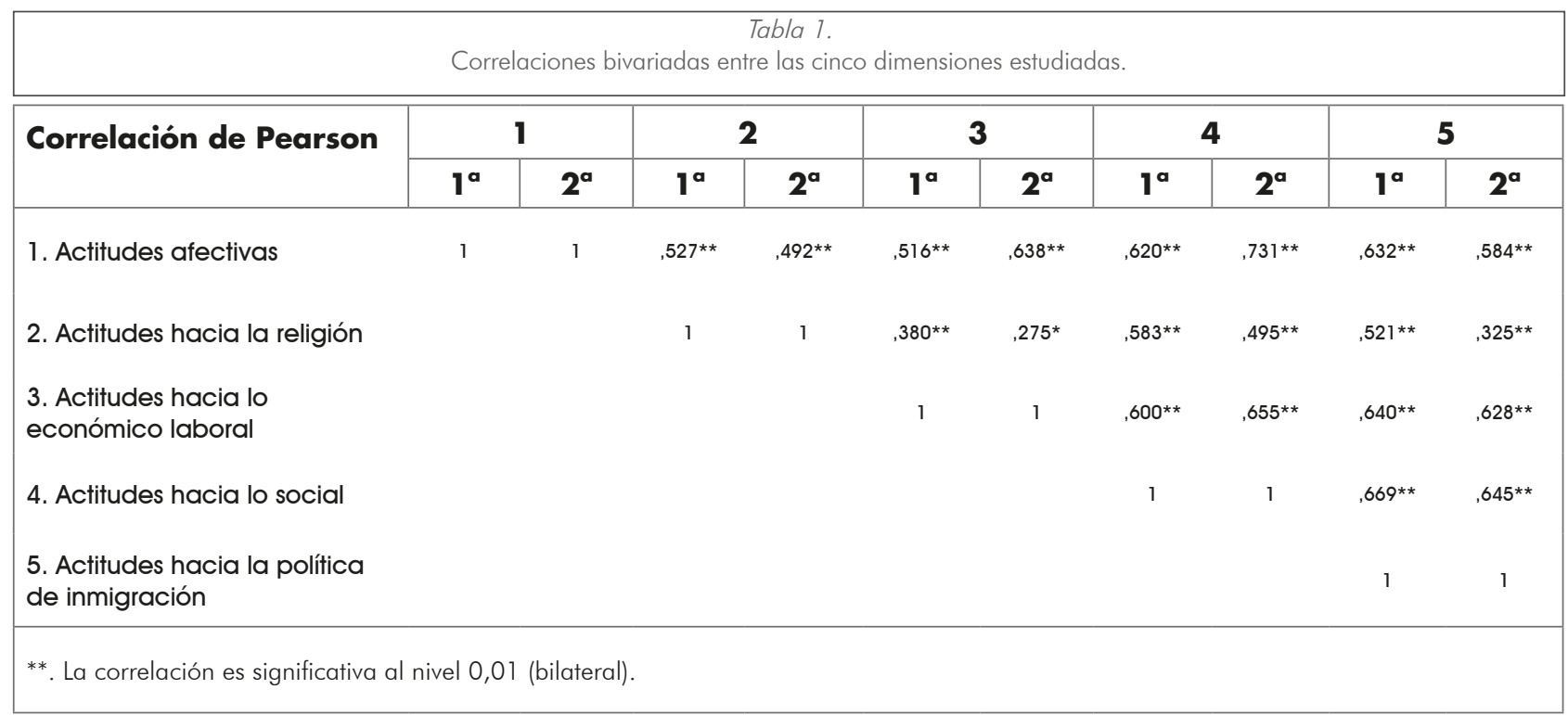

\begin{tabular}{|c|c|c|c|c|c|c|c|c|c|c|c|}
\hline & Medidas de tenc & encia ce & $\begin{array}{r}T \\
\text { ral y dis }\end{array}$ & la 2. & las dim & siones & udiado & & & & \\
\hline & \multirow[b]{2}{*}{$\begin{array}{c}\text { Alfa de } \\
\text { Cronbach }\end{array}$} & \multicolumn{2}{|c|}{$M$} & \multicolumn{2}{|c|}{ DT. } & \multicolumn{2}{|c|}{ Var. } & \multicolumn{2}{|c|}{ Asim. } & \multicolumn{2}{|c|}{ Curt. } \\
\hline & & $1^{a}$ & $2^{a}$ & $1^{a}$ & $2^{a}$ & $1^{a}$ & $2^{a}$ & $1^{a}$ & $2^{a}$ & $1^{a}$ & $2^{a}$ \\
\hline Actitudes afectivas &, 737 & 4,027 & 4,012 & 0,6192 & 0,657 & 0,383 & 0,433 & 0,116 & $-0,221$ & $-0,999$ & $-0,966$ \\
\hline Actitudes hacia la religión & .688 & 3,194 & 3,371 & 0,760 & 0,629 & 0,579 & 0,397 & $-0,162$ & $-0,130$ & $-0,580$ & $-0,683$ \\
\hline $\begin{array}{l}\text { Actitudes hacia lo económico } \\
\text { laboral }\end{array}$ & ,666 & 3,422 & 3,445 & 0,607 & 0,640 & 0,369 & 0,410 & $-0,028$ & 0,026 & 0,464 & $-0,707$ \\
\hline Actitudes hacia lo social & ,793 & 3,817 & 3,909 & 0,628 & 0,575 & 0,395 & 0,331 & $-0,121$ & 0,322 & 0,533 & $-0,917$ \\
\hline $\begin{array}{l}\text { Actitudes hacia la política de } \\
\text { inmigración }\end{array}$ & 799 & 3,456 & 3,656 & ,7886 & 8100, & 0,622 & 0,656 & $-0,320$ & $-0,503$ & $-0,312$ & $-0,107$ \\
\hline
\end{tabular}


Los resultados del estadístico † de Student para comparar si los valores obtenidos de las medias presentan diferencias estadísticamente significativas en todas las dimensiones en relación al sexo, indican que en la dimensión "Religión", las mujeres presentan una puntuación estadísticamente significativa más alta que los varones con un buen tamaño del efecto $(t(77)=-3,072 ; p<0.05$; $d=, 74 ; \mathrm{DM}=0,540)$, al igual en la segunda vuelta las mujeres también puntúan más alto en la dimensión "Religión" $(t(77)=-2,802$; $p<0.05 ; d=0,65 ; \mathrm{DM}=0,390$ ) y además también presentan una puntuación más alta que los varones en "Política de Inmigración" $(t+77)=-2,553 ; p<0.05 ; d=0,58$; $\mathrm{DM}=0,460)$.

En cuanto a dimensión "Económico-laboral" no presentan resultados estadísticamente significativos, aunque los hombres aumentan las puntuaciones otorgadas y bajan ligeramente las de mujeres.

\section{ANÁLISIS DE LA INFLUENCIA DE LOS MEDIOS DE COMUNICACIÓN SOBRE LAS ACTITUDES HACIA LOS REFUGIADOS PRE-TEST Y POST-TEST.}

Se ha realizado un análisis de varianza (ANOVA) para conocer si la percepción personal de la frecuencia del uso de los medios de comunicación les ha influido de alguna manera en las respuestas a las cinco dimensiones estudiadas, tanto en los resultados obtenidos al aplicar el cuestionario antes de la intervención como en los resultados posteriores. Se ha aplicado la prueba comparaciones múltiples post hoc de Bonferroni para la comparación por pares. $\mathrm{Se}$ encuentran diferencias estadísticamente significativas entre el uso de algunos medios de comunicación y la mayor puntuación en algunas dimensiones, sobre todo en la primera aplicación del cuestionario.

En la primera aplicación del cuestionario los resultados con respecto a "Ver TV" muestran que existen diferencias estadísticamente significativas entre los que dicen que ven poco y la ven mucho, en la dimensión Económicolaboral, puntuando más alto los que manifiestan que la ven poco con un tamaño del efecto grande $(F=4,775 ; \mathrm{gl}=76$; $p<0.05, d=$,95; $D M=0,517$ ), y en la dimensión "Política de inmigración" existen diferencias estadísticamente significativas entre los que se inclinan hacia poco y mucho, puntuando más alto los que la ven poco, con un tamaño del efecto grande $(F=4,159$; $g l=76 ; p<0,05 ; d=, 91 ; \mathrm{DM}=0,658)$.

En cuanto a la "Lectura de prensa", los resultados señalan que existen diferencias estadísticamente significativas entre los que perciben que la leen nada y mucho, en la dimensión "Religión", puntuando más alto los que no la leen y el tamaño del efecto es muy grande $(F=5,879 ; g l=76 ; p<0,05$; $d=1,1 ; \mathrm{DM}=0,789)$; en la dimensión "Económico-laboral" existen diferencias estadísticamente significativas entre los que dicen leer la prensa nada y poco, puntuando más alto los que no la leen, siendo grande el tamaño del efecto ( $F=4,239 ; \mathrm{gl}=76 ; p<$ $0,05 ; d=0,97 ; \mathrm{DM}=0,601)$. En la dimensión "Política de inmigración" existen diferencias estadísticamente significativas entre los que dicen no leerla y que piensan que le dedican poco tiempo, puntuando más alto los que no la leen, y el tamaño del efecto es muy grande $(\mathrm{F}=7,291 ; \mathrm{gl}=76 ; p<0,05 ; d=1,2$; $\mathrm{DM}=0,963)$, y entre los que no la leen y los que dicen que ocupan mucho de su tiempo en leerla, puntuando más alto los que no la leen, siendo muy grande también el tamaño del efecto $(F=7,291 ; \mathrm{gl}=76 ; p<0,05$; $d=1,28 ; \mathrm{DM}=0,780$ ).

Con respecto a la consulta de webs de internet se observan diferencias estadísticamente significativas para la dimensión "Social" entre los que no las consultan nada y los que opinan que dedican mucho de su tiempo a consultarlas, puntuando más alto los que no las consultan, presentando un tamaño del efecto medio ( $F=3,608$; $g l=76 ; p<0,05 ; d=0,67 ; D M=0,291)$.

En cuanto al uso de Twitter para extraer información sobre los refugiados, los resultados señalan diferencias estadísticamente significativas para la dimensión "Social" entre los que no lo utilizan y los que asumen que lo utilizan poco, puntuando más alto los que lo utilizan poco, observando que el 
tamaño del efecto que resulta es enorme $(\mathrm{F}=5,835 ; \mathrm{gl}=76 ; p<0,05 ; d=1,57$; $\mathrm{DM}=0,598)$, y para la dimensión "Política de inmigración entre los que no lo utilizan y los que dicen utilizarlo poco, puntuando más alto los que no lo utilizan, siendo también enorme el tamaño del efecto $(F=5,733 ; \mathrm{gl}=76$; $p<0,05 ; d=1,47 ; \mathrm{DM}=-1,02)$, y al comparar entre los que opinan que lo utilizan poco y mucho, puntúan más alto los que lo utilizan mucho, siendo grande el tamaño del efecto $(F=5,733 ; g l=76 ; p<0,05$; $d=1,05 ; \mathrm{DM}=-0,788$ ).

No se encuentran diferencias estadísticamente significativas en cuanto al uso de Youtube y Facebook en esta primera aplicación del cuestionario.

En la segunda aplicación del cuestionario realizado tras la intervención, solo se encontraron diferencias estadísticamente significativas en cuanto a la percepción del tiempo que emplean en la lectura de prensa para la dimensión "Religión" entre los que no la leen y los que manifiestan que leen mucho, puntuando más alto los que no la leen, presentando un tamaño del efecto medio $(F=3,981 ; g l=76 ; p<0,05 ; d=0,68$; $\mathrm{DM}=0,409$ ).

\section{DISCUSIÓN Y CONCLUSIONES}

Los objetivos de este trabajo eran, por un lado, comprobar si la realización de un ejercicio de empatía histórica sobre los exiliados de la Guerra Civil puede contribuir al desarrollo de actitudes tolerantes hacia la figura de los refugiados en el alumnado del Grado de Maestro en Educación Primaria, teniendo en cuenta las posibles diferencias en función del género, y por otro lado, verificar la influencia de los medios de comunicación en las actitudes de los mismos estudiantes hacia la figura de los refugiados y si estas se modificaban tras la práctica.

Los resultados indican una mejora general en la puntuación de las actitudes de los estudiantes hacia los refugiados, sobre todo en actitudes relacionadas con lo económicolaboral, lo social, la política de inmigración y la religión. En cuanto a diferencias por sexo, las mujeres manifiestan una mayor actitud positiva en las respuestas al segundo cuestionario en temas tan complejos como la religión o la política de inmigración. El resultado quizá deba relacionarse con un mayor número de fuentes consultadas por ellas a la hora de elaborar la práctica. Aunque las diferencias entre las respuestas a ambos cuestionarios no sean del todo llamativas, debe indicarse que al menos demuestran un cambio en las actitudes en un momento en el que lo ocurrido en París y Kabul estaba muy presente en los medios de comunicación y en la mentalidad colectiva.

No existen muchos estudios aún que midan el cambio de actitudes hacia determinados colectivos por medio de la Empatía Histórica. No obstante, el estudio corrobora que las experiencias educativas que aúnan la contextualización histórica y la comparación con situaciones similares y más cercanas al alumnado son muy eficaces a la hora de reducir prejuicios y estereotipos. El trabajo de Stefaniak y Bilewicz (2015) no solo tuvo como consecuencia que el alumnado entendiese la historia de los judíos polacos como propia, sino que también resultó en una mayor conciencia de igualdad con otro colectivo, el romaní. En este sentido, y teniendo en cuenta que algunos de los ítems de la dimensión "política de inmigración" trataban también el contexto de personas inmigrantes y no refugiadas, el aumento de las actitudes positivas indica la reducción de prejuicios con respecto a personas migrantes independientemente de su situación. Por lo tanto, el estudio indica que la empatía histórica puede ser una herramienta muy válida a la hora de la aproximación y comprensión desde el presente de situaciones del pasado, y desde el pasado a situaciones del presente que presenten similitudes, como es el caso de los desplazados por los conflictos bélicos. Aunque las redacciones elaboradas por los estudiantes en el papel de exiliados de la Guerra Civil Española estén siendo objeto de otro estudio, su contenido muestra un mayor interés en el tratamiento relacionado con temas concernientes a la causalidad en los casos en los que se utilizan un mayor número de fuentes históricas, tanto primarias como secundarias, lo que se encuentra en consonancia con los resultados obtenidos por Londoño y Carvajal (2016). 
En cuanto al uso de medios de comunicación como fuente de información sobre la situación de los refugiados, el estudio indica una influencia clara de los mismos en cuanto a las actitudes en el cuestionario previo. Los estudiantes que manifestaban tener actitudes más favorables a los refugiados en el cuestionario previo no utilizaban o utilizaban muy poco los medios tenidos en cuenta (televisión, prensa, Youtube, Internet, Twitter y Facebook) como fuente de información para acercarse a la figura de los refugiados. El resultado contradice en parte el estudio de Álvarez-Gálvez (2014b), que indicaba un menor uso de los medios informativos en las personas más reacias hacia el fenómeno de la inmigración. Hay que señalar que, en el cuestionario posterior a la práctica, los medios ya no influyen significativamente, excepto en el caso de la relación entre el consumo bajo de prensa y la dimensión religión existiendo actitudes más positivas entre los estudiantes que no la leen. Estos datos sugieren un desarrollo del pensamiento crítico del alumnado tras la recopilación y selección de distintas fuentes históricas para elaborar la práctica de los exiliados de la Guerra Civil Española.

Entre las limitaciones al presente estudio debe destacarse la carencia de grupo de control a la hora de llevar a cabo la experiencia, por lo que podría considerarse como un estudio pre-experimental y sometido a amenaza contra la validez interna. La experiencia fue realizada con un grupo-clase concreto, por lo que la selección de los participantes no fue aleatoria, a lo que debe sumarse el escaso tamaño de la muestra. Debe señalarse también el componente grupal de la experiencia. Se decidió medir el posible cambio a nivel grupal y no individual, lo que proporcionaría más datos a la hora de corroborar la viabilidad de este tipo de experiencias con el objeto de reducir prejuicios y estereotipos en cuanto a determinados colectivos sociales. Como línea de continuación y mejora, sería preciso llevar a cabo la experiencia con un grupo de control y otro experimental, midiendo los resultados de sujeto a sujeto, para comprobar si hay una mejora más significativa. Incluso tendría que implementarse tomando como base otras situaciones actuales que puedan relacionarse con hechos históricos distintos. También se hace necesario indagar más profundamente en el uso y frecuencia de los medios de comunicación y su relación con prejuicios y estereotipos hacia determinados colectivos.

\section{Conflicto de intereses}

Los autores declaran no tener ningún conflicto de intereses.

\section{REFERENCIAS}

Álvarez-Gálvez, J. (2014a). Television news consumption and public attitudes towards immigration: a multi-level approach. Revista de Psicología Social, 29(3), 401-429. doi: 10.1080/02134748.2014.972704

Álvarez-Gálvez, J. (2014b). El consumo de medios informativos y las actitudes hacia los inmigrantes. Revista Española de Investigaciones Sociológicas, 145(1), 3-24. doi: 10.5477/cis/reis. 145.3

Andreu, B. (2016). Movimientos migratorios e identidad. Historia, cultura y problemática abordada en las aulas desde la Historia Oral: el ejemplo del Sáhara Occidental. En R. García, A. Arroyo, y B. Andreu (Eds.), Deconstruir la alteridad desde la Didáctica de las Ciencias Sociales: Educar para una ciudadanía global (pp. 383-390). Madrid: AUPDCS.

Arnot, M., Pinson, H., y Candappa, M. (2009). Compassion, caring and justice: teachers' strategies to maintain moral integrity in the face of national hostility to the "non-citizen". Educational Review, 61(3), 249 264. doi: 10.1080/00131910903045906.

Barton, K. C., y Levstik, L. S. (2008). Teaching History for the common good. New Jersey: Taylor \& Francis.

Blinder, S. (2015). Imagined immigration: the impact of different meanings of immigrants in public opinion and policy debates in Britain. Political Studies, 63(1), 80-100. doi: 10.1111/1467-9248.12053.

Burgard, K., y Boucher, M. (2016). Same story; Different History: Students' racialized understanding of historic sites. The Urban Review, 48(5), 696-717.

Calderón, F., y Silva, C. (2014). A journey with a refugee family: raising culturally relevant teaching awareness. New Directions for Teaching and Learning, 140, 51-67. doi: 10.1002/t1.20114.

Carril, M. a T., y Sánchez, M. (2015). Visibilizando capacidades ocultas. Empatía y aprendizaje histórico en el alumnado de $1^{\circ}$ de la ESO. En A. M. ${ }^{a}$ Hernández, C. R. García, y J. L. de la Montaña (Eds.), Una enseñanza de las Ciencias Sociales para el futuro: recursos para trabajar la invisibilidad de personas, lugares y temáticas (pp. 669-677). Cáceres: AUPDCS.

Carril, M. a T., y Sánchez, M. (2016). Capacidad empática en profesores de Historia en formación: un estudio exploratorio. En R. López (Ed.), Ciencias Sociales, educación y futuro. Investigaciones en Didáctica de las Ciencias Sociales (pp. 150-161). Santiago de Compostela: Red 14. 
Cohen, J. (1988). Statistical power analysis for the behavioral science. Hillsdale, NJ: Lawrence Erlbaum Associates.

Domínguez, J. (2015). Pensamiento histórico y evaluación de competencias. Barcelona: GRAÓ.

Endacott, J. L. (2014). Negotiating the Process of Historical Empathy. Theory \& Research in Social Education, 42(1), 4-34. doi: 10.1080/00933104.2013.826158.

Erdfelder, E., Faul, F., y Buchner, A. (1996). G*POWER: a general power analysis program. Behavior Research Methods, Instruments, \& Computers, 28, 1-11.

Esses, V. M., Hamilton, L. K., y Gaucher D. (2017). The Global Refugee Crisis: Empirical Evidence and Policy Implications for Improving Public Attitudes and Facilitating Refugee Resettlement. Social Issues and Policy Review, 17(1), 78-123. doi: 10.1111/ sipr. 12028.

Etxeberría, F., Murua, H., Garmendia, J., y Etxeberría, J. (2013). Prejuicios, inmigración y educación. Actitudes del alumnado de secundaria en el País Vasco, Revista de Psicología y Educación, 8(1), 25-49.

Faas, D. (2011). The Nation, Europe and Migration. A comparison of Geography, History and Citizenship education curricula in Greece, Germany, and England. Journal of Curriculum Studies, 43(4), 471-492. doi: 10.1080/00220272.2011.584560.

Faul, F., Erdfelder, E., Lang, A. G., y Buchner, A. (2007). G*POWER 3: A flexible statistical power analysis program for the social, behavioral, and biomedical sciences. Behavior Research Methods, 39(2), 175-191.

Galikhuzina, R. G., Penkovtsev, R. V., y Shibanova, N. A. (2016). The image of refugees in the communication mass media: a source of conflict or cooperation? Academy of Marketing Studies Journal, 20(1), 27-32.

González, N., Henríquez, R., Pagès, J., y Santisteban, A. (2009). El aprendizaje de la empatía histórica en educación secundaria. Análisis y proyecciones de una investigación sobre la enseñanza y aprendizaje del conflicto y la convivencia en la Edad Media. En R. M. Ávila y I. Matozzi (coord.), La educación de la ciudadanía europea y la formación del profesorado. Un proyecto educativo para la estrategia de Lisboa (pp. 283-291). Bologna: AUPDCS.

Hartmann, U., y Hasselhorn, M. (2008). Historical perspective taking: A standarized measure for an aspect of students' historical thinking. Learning and Individual Differences, 18(2), 264-270.

Hope, J. (2008). "One day we had to run": the development of the refugee identity in children's literature and its function in education. Children's Literatures in Education, 39(4), 295-304. doi: 10.1007/s10583-008-9072-x.

Huigien, T., Boxtel, C., Grift, W., y Holthuis, P. (2014). Testing elementary and secondary school students' ability to perform historical perspective taking: the constructing of valid and reliable measure instruments. European Journal of Psycology of Education, 29(4), 653-672. doi: 10.1007/s10212-014-0219-4.
IBM Corp. Released 2013. IBM SPSS Statistics for Windows, Version 22.0. Armonk, NY: IBM Corp.

Isik-Ercan, Z. (2012). In pursuit of a new perspective in the education of children of the refugees: advocacy for the "family". Educational Sciences: Theory and Practice, 12(4), 3025-3038.

Lee, P., y Ashby, R. (2001). Empathy, perspective taking and rational understanding. En O. L. Davis, E. A. Yeager, y S. J. Foster (Eds.), Historical empathy and perspective taking in the social studies (pp. 21 -50). Oxford: Rowman \& Littlefield. Lévesque, S. (2008). Thinking Historically. Educating Students for the 21th Century. Toronto: University of Toronto Press.

Londoño, J. G., y Carvajal, J. P. (2016). Pedagogías para la memoria histórica: reflexiones y consideraciones para un proceso de innovación en el aula. Educación y Ciudad, 30(1), 65-78. doi: 10.14483/udistrital.jour. cpaz.2015.1.a07.

Monobe, G., y Son, E. H. (2014). Using children's literature and drama to explore children's lives in the context of global conflicts. The Social Studies, 105, 69-74. doi: 10.1080/00377996.2013.820164.

Navarro, E. (2015). El desarrollo de las competencias ciudadanas a través de la enseñanza de la Historia de España. Enseñanza de las Ciencias Sociales, 14, 49-59.

Rantala, J., Manninen, M., y Berg, M. (2016). Stepping into other people's shoes proves to be a difficult task for high school students: assessing historical empathy through simulation exercise. Journal of Curriculum Studies, 48(3), 323-345. doi: 10.1080/00220272.2015.1122092.

Roxas, K. (2010). Who really wants "the tired, the poor, and the huddle masses" anyway? Teachers' use of cultural scripts with refugee students in public schools. Multicultural Perspectives, 12(1), 65-73. doi: 10.1080/15210960.2010.481180.

Rutland, A., y Killen, M. (2015). A development science approach to reducing prejudice and social exclusion: Intergroup processes, social-cognitive development, and moral reasoning. Social Issues and Policy Review, 9(1), 121-154. doi: 10.1111/ sipr. 12012.

Sáiz, J. (2013). Empatía histórica, historia social e identidades: pensar históricamente la conquista cristiana de la Valencia musulmana con estudiantes de $2^{\circ}$ de ESO. Clío, 39. Disponible en http://clio.rediris.es/n39/articulos/historiasocial/ saiz.pdf

- Fecha de consulta 08/05/2017.

San Pedro, M. a B., y López, I. (2016). Relacionar pasado y presente mediante uso de fuentes y la empatía histórica: investigación con profesorado en formación. En R. López (Ed.), Ciencias Sociales, educación y futuro. Investigaciones en didáctica de las Ciencias Sociales (pp. 458-467). Santiago de Compostela: Red 14.

Santisteban, A. (2010). La formación de competencias de pensamiento histórico. Clío \& Asociados, 14, 34-56. 
Santisteban, A. (2015). La formación del profesorado para hacer visible lo invisible. En A. M. ${ }^{a}$ Hernández, C. R. García, y J. D. de la Montaña (Eds.). Una enseñanza de las Ciencias Sociales para el futuro: recursos para trabajar la invisibilidad de personas, lugares y temáticas (pp. 383-393). Cáceres: AUPDCS.

Santisteban, A., Tosar, B., Izquierdo, A., Llusà, J., Canals, R., González, N., y Pagès, J. (2016). La literacidad crítica de la información sobre los refugiados y refugiadas: construyendo la ciudadanía global desde la enseñanza de las Ciencias Sociales. En R. García, A. Arroyo, y B. Andreu (Eds.), Deconstruir la alteridad desde la Didáctica de las Ciencias Sociales: Educar para una ciudadanía global (pp. 550-560). Madrid: AUPDCS.

Seixas, P. (1996). Conceptualizing the growth of historical understanding. En D. R. Olson, y N. Torrance (Eds.), The handbook of education and human development (pp. 765-783). Oxford: Blackwell 765783.

Shemilt, D. (1984). Beauty and the philosopher: Empathy in history and classroom. En A. Dickinson, P. Lee, y P. Rogers (Eds.), Learning history (pp.39-84). Londres: Heinemann Educational Books.

Stefaniak, A., y Bilewicz, M. (2015). Contact with a multicultural past: a prejudice-reducing intervention. International Journal of Intercultural Relations, 50, 60-65. doi: 10.1016/i.iiintrel.2015.11.004.
Szilassy, E., y Árendás, Z. (2007). Understanding of "difference" in the speech of teachers dealing with refugee children in Hungary. Journal of Ethnic and Migration Studies, 33(3), 249-264. doi: 10.1080/13691830701234525.

Trepat, C.-A. (1995). Procedimientos en Historia. Un punto de vista didáctico. Barcelona: GRAÓ.

Valls, R. (2009). Ciudadanía y multiculturalidad en la enseñanza de las Ciencias Sociales: el desajuste entre intenciones educativas y prácticas escolares. En R. M. a Ávila, B. Borghi, y I. Matozzi (Eds.). La educación de la ciudadanía y la formación del profesorado. Un proyecto educativo para la "Estrategia de Lisboa" (pp. 109-115). Bologna: AUPDCS.

Wineburg, S. (2001). Historical Thinking and Other Unnaturals Acts: Charting the Future of Teaching the Past. Philadelphia: Temple University Press.

Yang, S.-C., y Huang, L. J. (2011). Historical thinking development of students: Applying the historical perspective-taking model. Journal of Research in Education Sciences, 56(4), 129-153.

Zembylas, M. (2012). The affective (re)production of refugee representations through educational policies and practices: reconceptualising the role of emotion for peace education in a divided country. International Review of Education, 58(4), 465-480. doi: 10.1007/ s11159-012-9296-2.
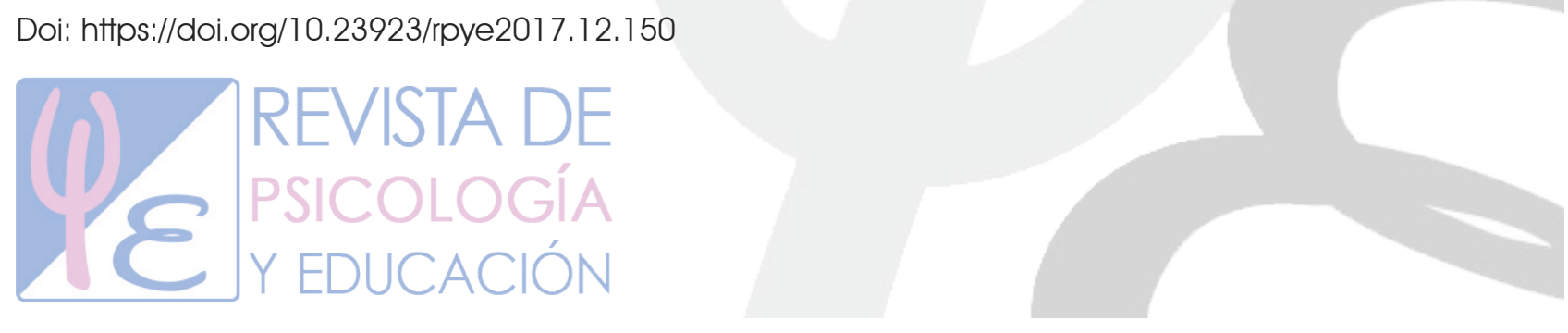